\title{
RELATIONSHIP OF THE ASY-H INDEX WITH INTERPLANETARY MEDIUM PARAMETERS AND AURORAL ACTIVITY IN MAGNETIC STORM MAIN PHASES DURING CIR AND ICME EVENTS
}

\author{
R.N. Boroyev \\ Yu.G. Shafer Institute of Cosmophysical Research and Aeronomy \\ of SB RAS, \\ Yakutsk,Russia,boroyev@ikfia.ysn.ru \\ Ammosov North-Eastern Federal University, \\ Yakutsk, Russia,boroyev@ikfia.ysn.ru
}

\author{
M.S. Vasiliev \\ Yu.G. Shafer Institute of Cosmophysical Research and Aeronomy \\ of SB RAS, \\ Yakutsk, Russia,ms_vasiliev@ikfia.ysn.ru \\ Ammosov North-Eastern Federal University, \\ Yakutsk, Russia,ms_vasiliev@ikfia.ysn.ru
}

\begin{abstract}
In this study, we examine the relationship of the $A S Y-H$ index characterizing the partial ring current intensity with interplanetary medium parameters and auroral activity during the main phase of magnetic storms, induced by the solar wind (SW) of different types. Over the period 1979-2017, 107 magnetic storms driven by CIR and ICME (MC + Ejecta) events have been selected. We consider magnetic storms with $D s t_{\min } \leq-50 \mathrm{nT}$. The average $A S Y-H$ index $\left(A S Y_{\text {aver }}\right)$ during the magnetic storm main phase is shown to increase with increasing SW electric field and southward IMF $B_{z}$ regardless of SW type. There is no relationship between $A S Y_{\text {aver }}$ and SW velocity. For the CIR and ICME events, the average $A E\left(A E_{\text {aver }}\right)$ and $K_{\mathrm{p}}\left(K_{\mathrm{p} \text { aver }}\right)$ indices have
\end{abstract}

been found to correlate with $A S Y_{\text {aver }}$. The highest correlation coefficient between $A E_{\text {aver }}$ and $A S Y_{\text {aver }}(r=0.74)$ is observed for the magnetic storms generated by CIR events. A closer relationship between $K_{\mathrm{p} \text { aver }}$ and $A S Y_{\text {aver }}$ $(r=0.64)$ is observed for the magnetic storms induced by ICME events. The $A S Y_{\text {aver }}$ variations correlate with $D s t_{\text {min }}$. The relationship between $A S Y_{\text {aver }}$ and the rate of storm development is weak.

Keywords: magnetic storm, $A S Y-H$ index, Dst index, solar wind, electric field.

\section{INTRODUCTION}

It is known that during magnetic storms large-scale magnetospheric current systems are enhanced, auroral electrojets shift to lower latitudes, and intense substorm disturbances occur. Simultaneously there is a significant decrease in the horizontal magnetic field component at low latitudes. Overall, geomagnetic indices vary considerably [Akasofu, Chapman, 1974; Nishida, 1980]. The low-latitude Dst index is used to evaluate the ring current intensity during magnetic storms and is a measure of geoeffectiveness of interplanetary disturbances [Sugiura, 1964; Burton et al., 1975]. The high-latitude $A E$ index and the mid-latitude $K_{\mathrm{p}}$ index characterize the auroral current intensity during magnetic storms and are indicators of substorm activity [Davis, Sugiura, 1966]. During a magnetic storm, the geomagnetic activity indices correlate [Lyatsky, Maltsev, 1983]. Development of the ring current is, however, not associated with substorms. According to [Iyemori, Rao, 1996; Sharma et al., 2003], the main cause of ring current development is the enhancement of magnetospheric convection during periods of the long-term southward IMF $B_{z}$ component whose efficiency is attributed to the SW electric field effect: $E_{\mathrm{sw}}=V_{\mathrm{sw}} \times B_{\mathrm{z}}$ [Gonzalez et al., 1994; Kane, 2005]. Among IMF (IMF $B, B_{z}$ ) and SW (velocity, plasma density and temperature) parameters, the electric field associated with SW plasma motion and southward $B_{z}$ is the main factor in the development of a magnetic storm [Gonzalez et al., 1994; Kane, 2005].

Results of statistical and morphological studies show that the intensity of magnetospheric-ionospheric dis- turbances (magnetic storms and substorms) also depends on SW type [Borovsky, Denton, 2006; Despirak et al., 2009]. At present, the following SW types are distinguished: interplanetary coronal mass ejections (ICME) comprising magnetic clouds (MC) and ejecta, corotating interaction regions (CIR), and compression areas before ICME (sheath). Each SW type has a specific set of SW and IMF parameters. Dremukhina et al. [2018] have examined coupling functions representing expressions for the SW electric field $E_{\mathrm{sw}}$, calculated through the SW velocity $V_{\mathrm{sw}}$ and magnetic field $B$ with regard to the hour angle and the plasma dynamic pressure effect. The use of the Barton coupling function relating the $\mathrm{SW}$ integral electric field $E_{\mathrm{sw}}$ to $D s t$ has been shown to provide the highest correlation coefficients for all SW types. The highest geoeffectiveness is observed for the sheath and CIR driven magnetic storms, whereas the ICME induced storms (MC+Ejecta) exhibit $D s t$ minimum in modulus $\left|D s t_{\min }\right|$ for large values of $E_{\mathrm{sw}}$ [Plotnikov, Barkova, 2007; Nikolaeva et al., 2011; Yermolaev et al., 2012]. The $A E$ index, unlike Dst, during the magnetic storm main phase does not depend on $E_{\mathrm{sw}}$ for almost all SW types except MC. There is a nonlinear dependence of $A E$ on $E_{\mathrm{sw}}$ in MC. The relationship between $K_{\mathrm{p}}$ and $E_{\mathrm{sw}}$ is characterized by a linear empirical dependence for CIR and a nonmonotonic dependence for MC [Plotnikov, Barkova, 2007; Yermolaev et al., 2012]. Boroyev and Vasiliev [2018] have, however, shown that for CIR, unlike ICME, $A E$ increases with increasing SW electric field. The $K_{\mathrm{p}}$ index correlates with $E_{\mathrm{sw}}$ only for ICME. The 
difference between the results is likely to arise from different methods of determining auroral activity during the magnetic storm main phase. The papers [Plotnikov, Barkova, 2007; Nikolaeva et al., 2011; Yermolaev et al., 2012] have compared extreme Dst, $A E$, and $K_{\mathrm{p}}$ with minimum $B_{z}$ and $E_{\mathrm{sw}}$ or $D s t_{\mathrm{min}}, A E$ and $K_{\mathrm{p}}$ with $B_{z}\left(E_{\mathrm{sw}}\right)$ for $D s t_{\text {min }}$. These approaches compare only individual (extreme) points during development of a process and weakly account for the dynamics of magnetic storm generation. Boroyev and Vasiliev [2018] have examined average $A E$ and $K_{\mathrm{p}}$ during the magnetic storm main phase and compared them with the average SW electric field.

During a magnetic storm, in addition to global current systems small-scale local current systems such as the partial ring current are formed which contribute greatly to Dst variations [Fok et al., 2001; Liemohn et al., 2001; Kozyra, Liemohn, 2003]. Unlike the symmetric ring current, the partial ring current is related to auroral currents through the system of field-aligned currents of the evening-night sector of the magnetosphere [Grafe et al., 1997; Feldstein et al., 2005; Barkhatova, 2013]. The occurrence of the partial ring current, as in the case of the symmetric ring current, is attributed to the injection of plasma particles into the inner magnetosphere during a magnetospheric convection enhancement [Bakhmina, Kalegaev, 2008; Kalegaev et al., 2008]. The partial ring current intensity is estimated using the average $A S Y-H$ index [http://wdc. kugi.kyoto-u.ac.jp]. Of particular interest are the studies of the relationship of the dynamics of the partial ring current with auroral disturbances and symmetric ring current during magnetic storms induced by SW of different types.

The purpose of this work is to examine the correlation relationships of $A S Y-H$ with interplanetary medium parameters and substorm activity indices during the main phase of magnetic storms induced by the solar wind of different types.

\section{EXPERIMENTAL DATA}

We estimate geomagnetic activity in this work, using the $A E, K_{\mathrm{p}}, A S Y-H$, and $D s t$ indices [http://swdcwww.kugi. kyoto-u.ac.jp/index.html]. Over the period from 1979 to 2017, 107 CIR and ICME induced magnetic storms (MC+Ejecta) with $D s t_{\min } \leq-50 \mathrm{nT}$ have been selected. We do not address other SW types here. A magnetic storm is considered to be related to SW of a given type if the main phase and the minimum Dst coincide in time with SW of this type. The method of classifying SW types is described in detail in [Yermolaev et al., 2009; Yermolaev et al., 2010]. On the website [ftp.iki.rssi.ru/pub/omni/catalog] is a catalog of SW types. For each event, as in [Boroyev, Vasiliev, 2018], we calculate average $A E, K_{\mathrm{p}}$, and $A S Y-H$ and the rate of magnetic storm development $(|\Delta D s t| / \Delta T)$ in the main phase.

Duration $\Delta T$ of the magnetic storm main phase was defined as an interval from the beginning of a sharp decrease in $D s t$ ( $D s t_{0}$ - value at this point) to the moment of recording of $D s t_{\min } ;|\Delta D s t|=\mid D s t_{\min }-$ $D s t_{0} \mid$. To account for the SW and IMF parameters, hourly average data [http://www.omniweb.com] is used to determine average values of the SW azimuth electric field, southward $B_{z}$, and SW velocity for a period coinciding with the magnetic storm main phase. The average interplanetary medium parameters and geomagnetic activity indices in general allow us to assess the development of the magnetic storm main phase.

To identify the relationship between the geomagnetic indices and the SW and IMF parameters, we utilize a linear approximation as the simplest way to establish the relationship between the values. We calculate Pearson correlation coefficients and probabilities of determining statistical significance.

\section{RESULTS}

Figure 1 shows the relationship between the average $A S Y-H A S Y_{\text {aver }}$ and the average SW electric field $E_{\text {sw aver }}$ in the main phases of CIR and ICME driven storms. Table 1 lists equations of linear regression between $A S Y_{\text {aver }}$ and $E_{\mathrm{sw}}$ aver, as well as correlation coefficients $r$ and significance level $P$. Figure 1 shows that during the magnetic storm main phase $A S Y_{\text {aver }}$ increases with $E_{\text {sw aver }}$ for both CIR and ICME. Consequently, the SW type is not reflected in $A S Y-H$ variations.

The azimuth electric field $E_{\mathrm{sw}}=V_{\mathrm{sw}} \times B_{z}$ is known to be associated with one of the significant geoeffective SW parameters - the southward IMF $B_{z}$ component [Gonzalez et al., 1994; Kane, 2005]. With SW type considered, we have made a correlation analysis of $A S Y-H$, southward $B_{z}$, and SW velocity $V_{\text {sw. }}$.

Figure 2, $a, c$ shows the average $A S Y$-Has a function of the average modulus of southward $B_{z}\left|B_{\text {zaver }}\right|$ in the main phases of magnetic storms for SW of two types; Table 2 lists $r, P$, and equations of linear regression between $A S Y_{\text {aver }}$ and $\left|B_{z \text { aver }}\right|$. Figure 2, $a, c$ indicates that for $\mathrm{SW}$ of both the types $A S Y_{\text {aver }}$ increases linearly with $\left|B_{z \text { aver }}\right|$ ( $r=0.63$ for CIR and $r=0.56$ for ICME). For both CIR and ICME there is, however, no clear linear relationship between $A S Y_{\text {aver }}$ and $V_{\text {sw aver }}$ (see Figure 2, $b, d$; correlation coefficients are omitted).

The relationship of $A S Y-H$ with $A E$ and $K_{\mathrm{p}}$ is shown in Figure 3. Table 3 presents $r, P$, and equations of linear regression between $A E_{\mathrm{aver}}, K_{\mathrm{p} \text { aver }}$, and $A S Y_{\text {aver }}$ in the magnetic storm main phases for SW of two types.
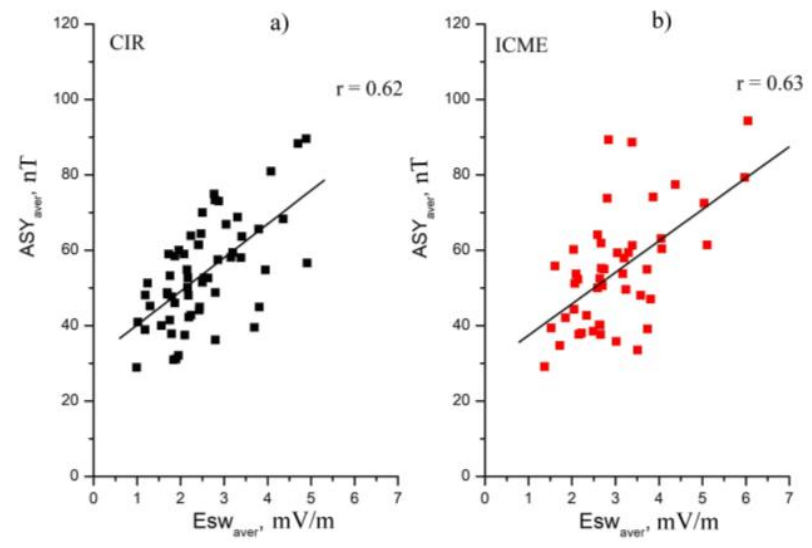

Figure 1. Average $A S Y-H\left(A S Y_{\text {aver }}\right)$ as a function of the average SW electric field ( $\left.E_{\mathrm{Sw} \text { aver }}\right)$ in the main phases of CIR and ICME induced magnetic storms: squares are individual magnetic storms; straight lines indicate a linear approximation 

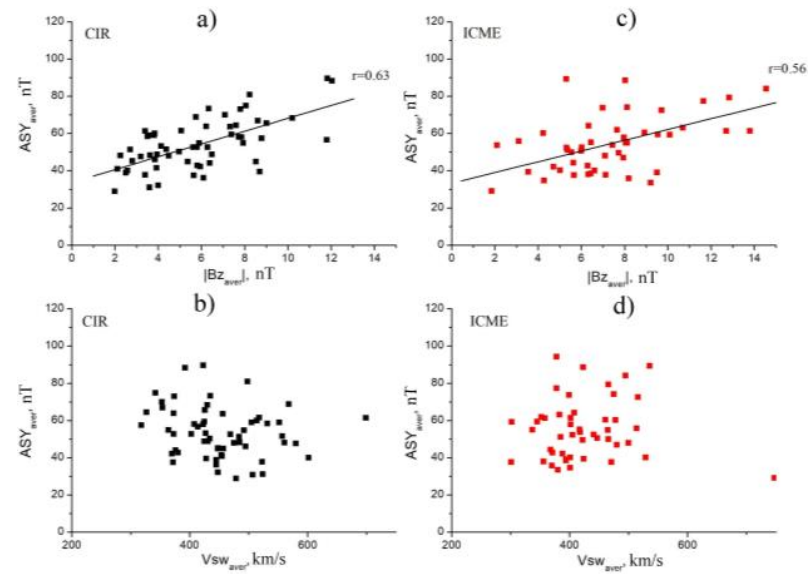

Figure 2. $A S Y_{\text {aver }}$ as a function of average values of the southward $B_{\mathrm{Z}}$ modulus and SW velocity in the main phases of CIR and ICME induced magnetic storms: squares are individual magnetic storms; straight lines indicate a linear approximation
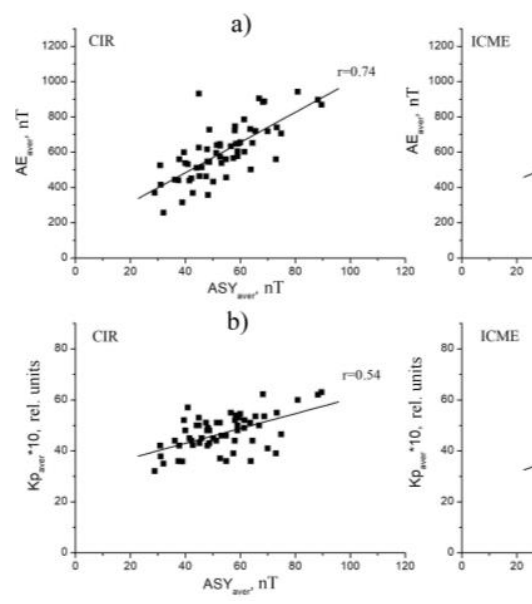

c)

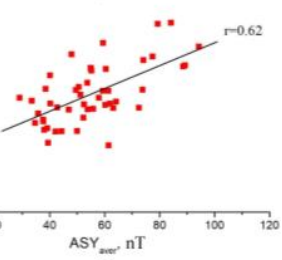

d)

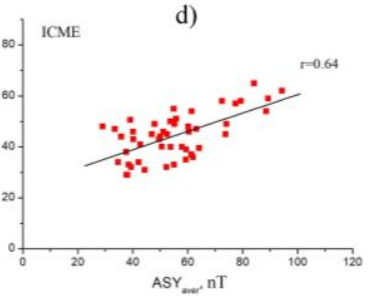

Figure 3. $A E_{\text {aver }}, K_{\mathrm{p} \text { aver }}$ versus $A S Y_{\text {aver }}$ in the main phases of CIR and ICME induced magnetic storms: squares are individual magnetic storms; straight lines indicate a linear approximation

Table 1

The number of magnetic storms $N$, correlation coefficients $r$, significance levels $P$, and equations of linear regression between $A S Y_{\mathrm{aver}}$ and $E_{\mathrm{sw} \text { aver }}$ for CIR and ICME

\begin{tabular}{|l|c|c|c|c|}
\hline \multirow{2}{*}{ SW type } & \multirow{2}{*}{$N$} & \multicolumn{3}{|c|}{$E_{\text {sw aver }}$} \\
\cline { 3 - 5 } & $N$ & $r$ & $P$ & approximation \\
\hline CIR & 59 & 0.62 & 0.99 & $y=8.9 x+31.2$ \\
\hline ICME & 48 & 0.63 & 0.99 & $y=8.3 x+29$ \\
\hline
\end{tabular}

Table 2

The number of magnetic storms $N$, correlation coefficients $r$, significance levels $P$, and equations of linear regression between $A S Y_{\text {aver }}$ and $\left|B_{z \text { aver }}\right|$ for CIR and ICME

\begin{tabular}{|l|c|c|c|c|}
\hline \multirow{2}{*}{ SW type } & \multirow{2}{*}{$N$} & \multicolumn{3}{|c|}{$\mid B_{z \text { aver }}$} \\
\cline { 3 - 5 } & & $r$ & $P$ & approximation \\
\hline CIR & 59 & 0.63 & 0.99 & $y=3.44 x+33.8$ \\
\hline ICME & 48 & 0.56 & 0.99 & $y=2.89 x+33.3$ \\
\hline
\end{tabular}

Figure 3, $a, c$ shows that during the magnetic storm main phase average $A E$ increases with average $A S Y-H$ for both CIR and ICME, but for CIR (Table 3) the correlation between $A E_{\text {aver }}$ and $A S Y_{\text {aver }}$ is higher $(r=0.74)$.
High correlation coefficients between $K_{\mathrm{p} \text { aver }}$ and $A S Y_{\text {aver }}$ (Figure 3, $e$ ) are observed for ICME $(r=0.64)$.

To elucidate the role of the partial ring current in developing low-latitude geomagnetic disturbances, we have performed a comparative analysis of $A S Y-H$ and $D s t_{\text {min }}$, as well as the rate of development of the magnetic storm main phase for SW of two types.

Figure 4, $a, c$ plots average $A S Y-H$ as a function of $\left|D s t_{\min }\right|$ for CIR and ICME driven magnetic storms. $A S Y_{\text {aver }}$ as a function of the rate of development of the magnetic storm main phase $|\Delta D s t| / \Delta T$ for $\mathrm{SW}$ of two types is presented in Figure 4, $b, d$. Figure $4, a, c$ indicates that in the magnetic storm main phase average $A S Y-H$ increases with $\left|D s t_{\min }\right|$ for $\mathrm{SW}$ of both the types, with a higher correlation coefficient between $A S Y_{\text {aver }}$ and $\left|D s t_{\min }\right|$ (Table 4) observed for CIR $(r=0.71)$. The CIR and ICME induced magnetic storms (Figure 4, $b, d$ ) exhibit low correlation coefficients between $A S Y_{\text {aver }}$ and the rate of development of the main phase.

\section{DISCUSSION AND CONCLUSIONS}

The difference between SW and IMF parameters in $\mathrm{SW}$ types is known to reveal itself in $A E, K_{\mathrm{p}}$, and Dst variations. For example, in the case of CIR, unlike ICME, during the magnetic storm main phase there are high SW velocities and small southward $B_{z}$ (e.g., [Nikolaeva et al., 2011]).

The geomagnetic indices depend not only on the intensity of current systems related to the interplanetary medium parameters, but also on their position relative to the station whose data is used to calculate the indices. The position of auroral (ionospheric) current systems depends on the auroral oval size: the wider is the oval, the lower are the latitudes at which auroral currents are observed In turn, the auroral oval size depends largely on variations in the southward $B_{z}$ component: a decrease in IMF $B_{z}$ causes the oval to expand and its boundaries to shift to low latitudes. This effect is most pronounced on the nightside of the magnetosphere. Considerable changes in IMF $B_{\mathrm{z}}$ occur during the magnetic storm main phase. Boroyev and Vasiliev [2018] assume
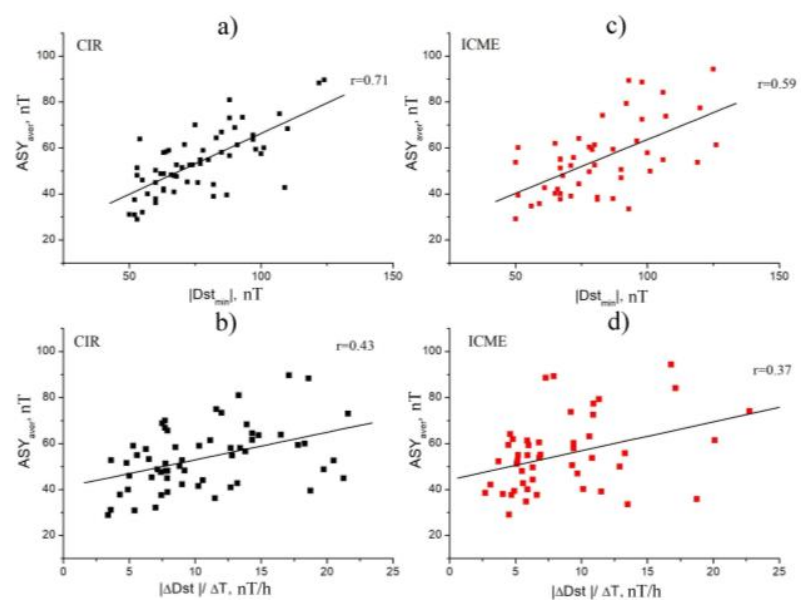

Figure 4. $A S Y_{\text {aver }}$ versus $\left|D s t_{\min }\right|$ and the rate of development of the main phases of CIR and ICME induced magnetic storms $|\Delta D s t| / \Delta T$ : squares are individual magnetic storms; straight lines indicate a linear approximation 
The number of magnetic storms $N$, correlation coefficients $r$, significance levels $P$, and equations of linear regression between $A E_{\text {aver }}, K_{\mathrm{p} \text { aver }}$, and $A S Y_{\text {aver }}$ for CIR and ICME

\begin{tabular}{|c|c|c|c|c|c|c|c|}
\hline \multirow[b]{2}{*}{ SW type } & \multirow[b]{2}{*}{$N$} & \multicolumn{3}{|c|}{$A E_{\text {aver }}$} & \multicolumn{3}{|c|}{$K_{\mathrm{p} \text { aver }}$} \\
\hline & & $r$ & $P$ & approximation & $r$ & $P$ & approximation \\
\hline CIR & 59 & 0.74 & 0.99 & $y=8.6 x+142$ & 0.54 & 0.99 & $y=0.29 x+31.4$ \\
\hline ICME & 48 & 0.62 & 0.99 & $y=6.5 x+312$ & 0.64 & 0.99 & $y=0.36 x+24.5$ \\
\hline
\end{tabular}

Table 4

The number of magnetic storms $N$, correlation coefficients $r$, significance levels $P$, and equations of linear regression between $A S Y_{\text {aver }}$ and $\left|D s t_{\min }\right|,|\Delta D s t| / \Delta T$ for CIR and ICME

\begin{tabular}{|l|c|c|c|c|c|c|c|}
\hline \multirow{2}{*}{ SW type } & $N$ & \multicolumn{4}{|c|}{$\left|D s t_{\text {min }}\right|$} & \multicolumn{3}{|c|}{$\mid \Delta$ Dst $\mid / \Delta T$} \\
\cline { 3 - 8 } & & $r$ & $P$ & approximation & $r$ & $P$ & approximation \\
\hline CIR & 59 & 0.71 & 0.99 & $y=0.5 x+13.5$ & 0.43 & 0.99 & $y=1.2 x+41$ \\
\hline ICME & 48 & 0.59 & 0.99 & $y=0.47 x+17$ & 0.37 & 0.99 & $y=1.3 x+44$ \\
\hline
\end{tabular}

that it is the SW type that determines the magnitude of shift of current systems relatively to the stations whose data is used to calculate the $A E$ and $K_{\mathrm{p}}$ indices. During ICME, unlike CIR, low values of the southward $B_{\mathrm{z}}$ component cause the auroral oval to expand and hence auroral currents to shift to lower latitudes. As a result, we can see higher correlation coefficients between $E_{\mathrm{sw}}$ aver and $K_{\mathrm{p} \text { aver }}$ than between $E_{\mathrm{sw} \text { aver }}$ and $A E_{\text {aver }}$.

The auroral activity indices $A E$ and $K_{\mathrm{p}}$ during a magnetic storm characterize the intensity of ionospheric currents. The question about the influence of SW type on magnetospheric currents remains open. In this paper, we have examined variations of the $A S Y-H$ index, which characterizes the intensity of the partial ring current, during CIR and ICME induced magnetic storms. According to some authors [Grafe et al., 1997; Feldstein et al., 2005; Barkhatov et al., 2008; Barkhatova, 2013], the partial ring current associated with auroral (ionospheric) currents is a magnetospheric part of the unified current system. For example, Barhatov et al. [2008] have assessed the role of magnetospheric-ionospheric current systems in the asymmetry of geomagnetic disturbance, as well as have examined the temporal dynamics of indices of partial ring current and auroral electrojets in the magnetic storm main phase regardless of SW type.

We, unlike [Barhatov et al., 2008], have carried out a correlation analysis of $A S Y-H$ and interplanetary medium parameters in the main phases of CIR and ICME induced magnetic storms, and have calculated average values of the indices and interplanetary medium parameters regardless of their temporal dynamics. The results confirm that the SW electric field and its related southward IMF $B_{z}$ component are the key geoeffective factors in the development of the partial ring current in the main phases of CIR and ICME driven magnetic storms. Figures 1, 2 suggest that in both the cases $A S Y-H$ depends on $E_{\mathrm{sw}}$ and IMF $B_{z}$ with high correlation coefficients $(r>0.5)$, but there is no clear relationship between $A S Y-H$ and SW velocity. SW type does not affect $A S Y$ $H$ variations during the magnetic storm main phase. Development of the magnetospheric current systems (partial ring current) probably does not depend on SW type. Dynamics of the partial ring current is completely determined by the magnetospheric convection depend- ing on the SW electric field. SW type has, however, no effect on the ionospheric part of the current system associated with the partial ring current. Figure 3 shows that $A S Y-H$ correlates with the intensity of auroral currents in the main phases of CIR and ICME induced magnetic storms. The highest correlation coefficient ( $r=0.74$ ) between the average values of $A E$ and $A S Y-H$ at a sufficiently high statistical significance $(P=0.99)$ is observed for CIR driven magnetic storms. On the contrary, a closer relationship between average $K_{\mathrm{p}}$ and $A S Y$ $H$ is observed for ICME induced magnetic storms. The difference between the $A E$ and $K_{\mathrm{p}}$ variations is likely to be due to the position of auroral electrojets relative to the stations whose data is used to calculate these indices [Boroyev, Vasiliev, 2018].

In this paper, we have also examined the relationship of the partial ring current with the intensity and rate of development of the main phases of CIR and ICME induced magnetic storms. Figure 4 indicates that the partial ring current has no significant effect on the rate of development of a magnetic storm. The calculations show low correlation coefficients between $A S Y-H$ and $|\Delta D s t| / \Delta T$ for CIR $(r=0.43)$ and ICME $(r=0.37)$. This is likely to be due to the fact that the partial ring current as a local current system can be observed in the magnetic storm main phase for only a few hours in contrast to large-scale magnetospheric current systems (current at the magnetopause symmetric ring current, magnetotail currents). Hence, the contribution of the partial ring current to the rate of development of a magnetic storm will be negligible.

The partial ring current, however, affects the Dst index. The analysis has shown that $A S Y-H$ correlates with $D s t_{\min }$ for CIR and ICME induced magnetic storms. These results agree with those obtain previously [Liemohn et al., 2001; Feldstein et al., 2005]. Thus, according to the model [Liemohn et al., 2001], during the magnetic storm main phase and early recovery phase the contribution of the asymmetric part of the ring current to $D s t$ variations predominates over the contribution of magnetotail currents. According to the estimates made by other authors [Feldstein et al., 2005], contributions of the partial ring current and magnetotail currents vary from 25 to $80 \%$. Thus, the partial ring current in the 
magnetic storm main phase further contributes to the dawn-dusk asymmetry of low-latitude geomagnetic disturbances [Love, Gannon, 2009].

In the future, we plan to analyze the ring current asymmetry in the main phases of magnetic storms induced by SW of different types, using satellite ENA observations and data from ground-based magnetometers.

We have obtained the following results:

1 . The average $A S Y-H$ index $\left(A S Y_{\text {aver }}\right)$ in the magnetic storm main phase depends on the SW electric field and the southward IMF $B_{z}$ componen. The SW type does not affect $A S Y_{\text {aver }}$ variations. There is no relationship between $A S Y_{\text {aver }}$ and SW velocity.

2. The average $A E\left(A E_{\text {aver }}\right)$ and $K_{\mathrm{p}}\left(K_{\mathrm{p} \text { aver }}\right)$ indices correlate with $A S Y_{\text {aver }}$ for both CIR and ICME. The highest correlation coefficient between $A E_{\text {aver }}$ and $A S Y_{\mathrm{a}-}$ ver $(r=0.74)$ is observed for the CIR driven magnetic storms, whereas a closer relationship between $K_{\mathrm{p} \text { aver }}$ and $A S Y_{\text {aver }}(r=0.64)$ is observed for the ICME induced magnetic storms.

3. $A S Y_{\text {aver }}$ correlates with minimum $D s t$ for both CIR and ICME. The relationship between $A S Y_{\text {aver }}$ and the rate of development of the magnetic storm main phase is weak.

The study was funded by RFBR under research project No. 20-05-00269 and partially under project AAAA-A17-11702145 0059-3.

\section{REFERENCES}

Akasofu S.-I., Chapman S. Solnechno-zemnaya fizika [Solar-Terrestrial Physics]. Moscow, Mir Publ., 1974, 384 p. (In Russian). (English edition: Akasofu S.-I., Chapman S. SolarTerrestrial Physics. Oxford, Clarendon Press, 1972, 901 p.).

Barkhatov N.A., Levitin A.E, Tserkovnyuk O.M. Relation of the indices characterizing the symmetric $(S Y M)$ and asymmetric $(A S Y)$ ring currents to the $A E(A U, A L)$ indices of auroral electrojet activity. Geomagnetizm i aeronomiya [Geomagnetism and Aeronomy]. 2008, vol. 48, no. 4, pp. 520-525. (In Russian).

Barkhatova O.M. Nonlinear connection between the auroral $(A U, A L)$ and mid-latitude $(S Y M-H, A S Y-H)$ geomagnetic activity indices at the main phase of geomagnetic storm. Solnechno-zemnaja fizika [Solar-Terrestrial Physics]. 2013 , vol. 23, pp. 100-108. (In Russian).

Bakhmina K.Yu., Kalegaev V.V. Modeling the partial ring current effect in a disturbed magnetosphere. Geomagnetizm $i$ aeronomiya [Geomagnetism and Aeronomy]. 2008, vol. 48, no. 6, pp. 770-779. (In Russian).

Borovsky J.E., Denton M.H. Differences between CME driven storms and CIR driven storms. J. Geophys. Res. 2006 , vol. 111. DOI: 10.1029/2005JA011447.

Boroyev R.N., Vasiliev M.S. Substorm activity during the main phase of magnetic storms induced by the CIR and ICME events. Adv. Space Res. 2018, vol. 61, iss. 1, pp. 348-354 DOI: 10.1016/j.asr.2017.10.031.

Burton R.K., McPherron R.L., Russell C.T. An empirical relationship between interplanetary conditions and Dst. J. Geophys. Res. 1975, vol. 80, iss. 31, pp. 4204-4214. DOI: 10.1029/JA080i031p04204.

Davis T.N., Sugiura M. Auroral electrojet activity index $A E$ and its universal time variations. J. Geophys. Res. 1966, vol. 71, iss. 3, pp. 785-801. DOI: 10.1029/JZ071i003p00785.

Despirak I.V., Lubchich A.A., Yahnin A.G., Kozelov B.V., Biernat H.K. Development of substorm bulges during different solar wind structures. Ann. Geophys. 2009, vol. 27, pp. 19511960. DOI: 10.5194/angeo-27-1951-2009.

Dremukhina L.A., Lodkina I.G., Yermolaev Y.I. Statistical study of the effect of different solar wind types on magnetic storm generation during 1995-2016. Geomagnetizm i aeronomiya [Geomagnetism and Aeronomy]. 2018, vol. 58, no.6, pp. 760-768. (In Russian). DOI: 10.1134/S0016794018060032.

Feldstein Y.I., Levitin A.E., Kozyra J.U., Tsurutani B.T., Prigancova A., Alperovich L., et al. Self-consistent modeling of the large-scale distortions in the geomagnetic field during the 24-27 September 1998 major magnetic storm. J. Geophys. Res. 2005, vol. 110. DOI: 10.1029/2004JA010584.

Fok M.-C., Wolf R.A., Spiro R.W., Moore T.E. Comprehensive computational model of the Earth's ring current. $J$. Geophys. Res. 2001, vol. 106, iss. A5, pp. 8417-8424. DOI: 10.1029/2000JA000235.

Gonzalez W.D., Joselyn J.A., Kamide Y., Kroehl H.W., Rostoker G., Tsurutani B.T., Vasyliunas V.M. What is a geomagnetic storm? J. Geophys. Res. 1994, vol. 99, pp. 57715792. DOI: 10.1029/93JA02867.

Grafe A., Bespalov P.A., Trakhtengerts V.Y., Demek-hov A.G. Afternoon mid-latitude current system and low-latitude geomagnetic field asymmetry during geomagnetic storms. Ann. Geophys. 1997, vol. 15, iss. 12, pp. 1537-1547. DOI: 10.1007/s00585-997-1537-5.

Iyemori T., Rao D.R.K. Decay of the Dst field of geomagnetic disturbance after substorm onset and its implication to storm-substorm relation. Ann. Geophys. 1996, vol. 14, iss. 6, pp. 618-622. DOI: 10.1007/s00585-996-0608-3.

Kane R.P. How good is the relationship of solar and interplanetary plasma parameters with geomagnetic storms? $J$. Geophys. Res. 2005, vol. 110, A022B. DOI: 10.1029/2004 JA010799.

Kalegaev V.V., Bakhmina K.Yu., Alexeev I.I., Belenkaya E.S., Feldstein Ya I., Ganushkina N.V. Ring current asymmetry during a magnetic storm. Geomagnetism and Aeronomy. 2008, vol. 48, no. 6, pp. 747-758. DOI: 10.1134/ S0016793208060078.

Kozyra J.U., Liemohn M.W. Ring current energy input and decay. Space Sci. Rev. 2003, vol. 109, pp. 105-131.

Liemohn M.W., Kozyra J.U., Thomsen M.F., Roeder J.L., Lu G., Borovsky J.E., Cayton T.E. Dominant role of the asymmetric ring current in producing the stormtime Dst. J. Geophys. Res. 2001, vol. 106, pp. 10883-10904. DOI: 10.1029/2000JA000326.

Love J.J., Gannon J.L. Revised Dst and the epicycles of magnetic disturbance: 1958-2007. Ann. Geophys. 2009, vol. 27 , iss. 8, pp. 3101-3131. DOI: 10.5194/angeo-27-3101-2009.

Lyatsky V.B., Maltsev Yu.P. Magnitosferno-ionosfernoye vzaimodeistvie [Magnetosphere-Ionosphere Coupling]. Moscow, Nauka Publ., 1983, 192 p. (In Russian).

Nikolaeva N.S., Yermolaev Y.I., Lodkina I.G. Dependence of geomagnetic activity during magnetic storms on the solar wind parameters for different types of streams. Geomagnetizm i aeronomiya [Geomagnetism and Aeronomy]. 2011, vol. 51, no.1, pp. 51-67. (In Russian).

Nishida A. Geomagnitnyi diagnoz magnitosfery [Geomagnetic Diagnosis of the Magnetosphere]. Moscow, Mir Publ., 1980, 299 p. (In Russian). (English edition: Nishida A. Geomagnetic Diagnosis of the Magnetosphere. New York, Heidelberg, Berlin, Springer-Verlag, 1978, 256 p.).

Plotnikov I.Ya., Barkova E.S. Advances in space research nonlinear dependence of $D s t$ and $A E$ indices on the electric field of magnetic clouds. Adv. Space Res. 2007, vol. 40, pp. 1858-1862. DOI: 10.1016/j.asr.2007.09.025.

Sharma A.S., Baker D.N., Grande M., Kamide Y., Lakhina G.S., McPherron R.M., Reeves G.D., Rostoker G., 
Vondrak R., Zelenyiio L. Storm-substorm relationship: Current understanding and outlook. Disturbances in Geospace: The Storm-Substorm Relationship. 2003, 268 p. (Geophys. Monogr. Ser., vol. 142). DOI: 10.1029/142GM01.

Sugiura M. Hourly values of the equatorial Dst for IGY. Annales of the International Geophysical Year. 1964, vol. 35, pp. 945-948.

Yermolaev Yu.I., Nikolaeva N.S., Lodkina I.G., Yermolaev M.Yu. Catalog of large-scale solar wind phenomena during 1976-2000. Kosmicheskiye issledovaniya [Cosmic Research]. 2009, vol. 47, no. 2, pp. 99-113. (In Russian).

Yermolaev Y.I., Nikolaeva N.S., Lodkina I.G., Yermolaev M.Yu. Specific interplanetary conditions for CIR-, Sheath-, and ICME-induced geomagnetic storms obtained by double superposed epoch analysis. Ann. Geophysicae. 2010, vol. 28, pp. 2177-2186. DOI: 10.5194/angeo-28-2177-2010.

Yermolaev Y.I., Nikolaeva N.S., Lodkina I.G., Yermolaev M.Y. Geoeffectiveness and efficiency of CIR, sheath, and ICME in generation of magnetic storms. J. Geophys. Res. 2012, vol. 117, A00L07. DOI: 10.1029/2011JA017139.

URL: http://wdc.kugi.kyoto-u.ac.jp (accessed 30 September 2019)

URL: http://swdcwww.kugi.kyoto-u.ac.jp/index.html (accessed 30 September 2019).

URL: ftp.iki.rssi.ru/pub/omni/catalog (accessed 30 September 2019)

URL: http://www.omniweb.com (accessed 30 September 2019).

How to cite this article

Boroyev R.N., Vasiliev M.S. Relationship of the ASY-H index with interplanetary medium parameters and auroral activity in magnetic storm main phases during CIR and ICME events. Solar-Terrestrial Physics. 2020. Vol. 6. Iss 1. P. 35-40. DOI: 10.12737/stp-61202004. 